\title{
Ontology-Based Representation and Reasoning Framework for Supporting Job Hazard Analysis
}

\author{
Han-Hsiang Wang, Ph.D. ${ }^{1}$; and Frank Boukamp, Ph.D., A.M.ASCE ${ }^{2}$
}

\begin{abstract}
Job hazard analysis (JHA) is a process of identifying potential hazards for each step of an activity and proposing safety rules to prevent potential incidents related to these hazards. In the United States, the Occupational Safety and Health Administration (OSHA) recommends performing JHA for construction activities to highlight and react to potential hazards. JHA commonly requires brainstorming sessions to identify steps and associated hazards within construction activities. A company's personnel involved in JHAs rely on their experience — and often also on the company's internal knowledge represented in the form of safety rules. However, the complexity and time-consuming nature of JHA prevent safety personnel from adjusting JHAs quickly when changes in the construction methods and the schedule are made. This paper presents a framework aiming to improve access to a company's JHA knowledge. The framework uses ontologies for structuring knowledge about activities, job steps, and hazards. It also includes an ontological reasoning mechanism for identifying safety rules applicable to given activities. The framework has been validated through several test cases using real JHA documents. The results of the validations are discussed, and conclusions for future research are drawn. DOI: 10.1061/(ASCE)CP.1943-5487.0000125. (C) 2011 American Society of Civil Engineers.
\end{abstract}

CE Database subject headings: Construction management; Occupational safety.

Author keywords: Ontology; Construction management; Safety; Job hazard analysis; Occupational Safety and Health Administration (OSHA).

\section{Introduction}

The construction industry is the industry with the highest potential for occupational hazard events in the United States. According to the statistics of the U.S. Bureau of Labor Statistics, the construction industry (including private sector, government, and self-employed workers) accounted for 1,282 out of 5,703 fatal work injuries recorded in 2006 - the most of any industry sector (U.S. Bureau of Labor Statistics 2007).

To prevent occupational hazards, many research efforts have been conducted to understand, identify, and analyze the trends and root causes of construction accidents (Abdelhamid and Everett 2000; Arboleda and Abraham 2004; Hinze et al. 1998). These research efforts provide important management information through qualitative posthazard analysis. This allows project participants to take precautions against eventual accident reoccurrence. Instead of relying on posthazard analysis, other research focused on analyzing construction activities or activity-related factors that may result in potential hazards, such as equipment or space requirements, with the goal of accident prevention through planning (Akinci 2000; Kim et al. 2006; Navon and Kolton 2007).

\footnotetext{
${ }^{1}$ Adjunct Assistant Professor, Dept. of Civil Engineering, National Chiao Tung Univ., 1001 University Rd., Hsinchu, 300, Taiwan. E-mail: hanhsiang.wang@gmail.com

${ }^{2}$ Senior Lecturer, School of Property, Construction and Project Management, Royal Melbourne Institute of Technology, GPO Box 2476, Melbourne, VIC 3001, Australia (corresponding author). E-mail: frank .boukamp@rmit.edu.au

Note. This manuscript was submitted on June 20, 2010; approved on April 13, 2011; published online on April 15, 2011. Discussion period open until April 1, 2012; separate discussions must be submitted for individual papers. This paper is part of the Journal of Computing in Civil Engineering, Vol. 25, No. 6, November 1, 2011. CASCE, ISSN 0887-3801/2011/6$442-456 / \$ 25.00$.
}

Among different construction safety practices, job hazard analysis (JHA) is one of the most prevalent safety management practices in the industry, according to certified safety professionals and experienced construction safety engineers (Roughton and Crutchfield 2007). The Occupational Safety and Health Administration (OSHA) recommends conducting JHA to address hazards in workplaces and to reduce worker injuries and illnesses (U.S. Department of Labor 2002). JHA is a process of identifying potential hazards for each step of an activity and proposing safety rules to eliminate or control these hazards.

The basic procedure for conducting a JHA includes (1) identifying all job steps of a given activity; (2) identifying hazards related to these different job steps; and (3) proposing safety rules (e.g., safety procedures or precautions) to eliminate, reduce, or control each hazard. The personnel conducting JHA rely on brainstorming sessions to identify steps within different construction activities and to identify the associated hazards. During the sessions, the involved personnel draw from their experience and often also from safety knowledge represented in the form of safety rules. Additionally, JHAs from previous activities may be revisited for guidance during a JHA for a new and similar activity. However, reviewing previous JHAs stored in knowledge bases is time-consuming when the number of activities and their respective steps and associated hazards becomes large. In addition, useful information about potential hazards and associated safety rules from job steps of previous JHAs may be missed if steps of the previous activity are identical to steps of the new activity but the previous activities fall into different categories than the new activities and thus are ignored.

Because of the complexity and time-consuming nature of JHA, safety personnel must perform JHAs often weeks, sometimes even months, before the activity actually is scheduled to be performed (Boukamp and Wang 2008). This makes it difficult to quickly react to changes in the construction plans and schedules while appropriately managing the resulting safety concerns. 
Therefore, this research proposes a framework that aims to improve access to a company's JHA knowledge. JHA knowledge is usually stored in JHA documents and includes two parts, JHA concepts and JHA safety rules, which are discussed in detail in the next section. The proposed framework represents JHA concepts in a concept ontology. The framework also includes an ontological reasoning mechanism that is developed to enable evaluating the concepts and to identify safety rules applicable to given conditions described through the concepts. The evaluation process can evaluate each safety rule's applicability, enabling classification of the safety rules according to their applicability.

This paper first provides an overview of JHA knowledge and discusses related research background. Then, the proposed framework is illustrated, which is followed by a discussion of case studies performed to demonstrate the advantages of the proposed framework and discussions on essential notions discovered in the case studies. The final section elaborates on how the framework can benefit the preparation of JHAs and on possible implications of the framework.

\section{Overview of JHA Knowledge}

JHA knowledge consists of JHA concepts and JHA safety rules and is usually stored in JHA documents (an example document is shown in Fig. 1). JHA concepts describe activities, job steps, and associated hazards, and JHA safety rules are rules imposed to address the identified hazards. Safety rules can be tied to a combination of hazard, job step, and activity concepts or to identified hazard concepts alone. JHA safety rules associated with the same set of JHA concepts comprise recommended safety procedures in JHA documents.

\section{Job Hazard Analysis Project: $\mathrm{ABC}$}

Contractor(s): XYZ Activity: Frame Column Work Plan Dated: April 5, 2007

\begin{tabular}{|c|c|c|}
\hline Job Steps & Potential Hazards & Recommended Safety Procedure \\
\hline $\begin{array}{l}\text { Fly forms to area } \\
\text { to be installed }\end{array}$ & Material dislodgement & $\begin{array}{l}\text {-Inspect rigging to be/being used } \\
\bullet \text { Ensure proper rigging method } \\
\text { Ensure direct contact with crane operator } \\
\text { - Clear area to land material } \\
\text { - Use taglines }\end{array}$ \\
\hline \multirow[t]{2}{*}{$\begin{array}{l}\text { Take forms off } \\
\text { cart/ blocking }\end{array}$} & Sprain/Strain of back & $\begin{array}{l}\text { Get assistance; Work with a partner. } \\
\text { Discuss lifting and moving process with } \\
\text { partner. }\end{array}$ \\
\hline & Slip/Trip/Fall & - Clear path from cart to work location. \\
\hline \multirow[t]{5}{*}{$\begin{array}{l}\text { Stand forms into } \\
\text { place }\end{array}$} & Sprain/Strain of back & $\begin{array}{l}\text { Use proper lifting technique. } \\
\text {-Get assistance; Work with a partner. }\end{array}$ \\
\hline & Pinched fingers & $\begin{array}{l}\text {-Wear slip resistant gloves. } \\
\text {-Set form on ground away from adjacent } \\
\text { form then grab form in a place where your } \\
\text { fingers will not get pinched. }\end{array}$ \\
\hline & Sharp edges & $\begin{array}{l}\text {-Review rebars conditions for sharp edges } \\
\text { or tie wire hazards. }\end{array}$ \\
\hline & Fall & $\begin{array}{l}\text { Use ladder or scaffold; do not use top } 2 \\
\text { rungs of ladder. }\end{array}$ \\
\hline & & $\begin{array}{l}\text { - Ensure area around ladder/ scaffold is } \\
\text { clear of debris and flat. }\end{array}$ \\
\hline \multirow[t]{4}{*}{ Set pins } & Fall & $\begin{array}{l}\text { - Use a portable ladder in the proper } \\
\text { manner. }\end{array}$ \\
\hline & & $\begin{array}{l}\text { - Get a partner to hold the form when } \\
\text { needed. }\end{array}$ \\
\hline & & $\begin{array}{l}\text {-Use scaffolding where possible; Scaffold } \\
\text { must be erected under supervision of a } \\
\text { competent person; All guardrails must be } \\
\text { installed and pins used; No substitute } \\
\text { materials! }\end{array}$ \\
\hline & & $\begin{array}{l}\text { If climbing form, must use retractable } \\
\text { lanyard anchored to top of form when feet } \\
\text { are higher than 6' off working surface. }\end{array}$ \\
\hline
\end{tabular}

Fig. 1. Example JHA document
A review of actual JHA documents showed that the three types of JHA concepts have the following semantic properties:

1. Activity concepts usually are division-oriented: it can be easily identified what construction divisions they belong to by looking at their titles. For example, activity concepts "pour columns" and "welding operation" can be easily categorized into the concrete and mechanical division respectively.

2. Job step concepts are specified through single- or multipleaction concepts that are usually stringed together with singleor multiple-resource concepts. Examples are "install piping overhead in lateral racks" and "drill and secure anchors and hangers."

3. Hazard concepts are unconditionally or conditionally specified through general or specific unsafe behavior, environments, injuries, or illnesses. For example, the hazard concept "injury to personnel due to hydraulic jack crushing" is a general injury tied to a condition, but the hazard concept "dermatitis" is a specific illness not tied to any condition.

These semantic properties provide guidance for how JHA concepts of the same type can be categorized. Therefore, they will be considered in the development of the framework discussed in the "Proposed Framework" section. In addition, four characteristics of JHA knowledge in a JHA document were identified:

1. JHA knowledge is hierarchically organized in a JHA document: an activity can comprise multiple job steps; a job step can have multiple associated hazards; a hazard must have a recommended safety procedure that can consist of multiple safety rules. Activity, job step, hazard, and recommended safety procedure become the four constituent elements in JHA documents.

2. JHA knowledge is hierarchically organized: it can be deemed to be composed of multiple hierarchical branches, consisting of activities, job steps, hazards, and recommended safety procedures; a recommended safety procedure can have a single or multiple JHA safety rules. For example, the first branch of the document in Fig. 1 is "frame columns," "fly forms to area to be installed," "material dislodgement" and contains five safety rules from "inspect rigging..." to "use taglines."

3. The activity, job step, and hazard in each branch represent the conditions in which the JHA knowledge in the branch applies. For example, the activity "frame columns", job step "fly forms to area to be installed" and hazard "material dislodgement" together describe the applicability condition in which these concepts' corresponding JHA safety rules applies.

4. Safety rules contained in the recommended safety procedure of each branch need to be put into practice when their corresponding applicability conditions are satisfied. For example, there are five safety rules in the first branch in the document in Fig. 1. These safety rules should be enforced to address the hazard "material dislodgement" when their applicability conditions described through the activity, job step, and hazard are satisfied.

These characteristics provide essential information about what can be leveraged when JHA documents are modeled to facilitate access to a company's JHA knowledge. Therefore, these characteristics are taken into account in the proposed framework.

\section{Research Background}

The research background targets four main topics related to this research. The first topic is document modeling, which is adopted in the proposed framework to model JHA documents. The second topic is extensible markup language (XML), which is used as a 
document modeling approach. The third topic is ontological modeling, which is leveraged to model JHA concepts in ontologies and build the proposed framework. The fourth topic is the web ontology language (OWL), which is the ontology language used to implement ontological modeling.

\section{Document Modeling}

Renear (2008) defined document modeling as "a principled systematic representation of textual information in order to improve the efficiency, functionality and interoperability of the creation, management, and exploitation of documents and document-like content." DeRose et al. (1990) summarized different document modeling approaches that deal with textual content of a document in different ways. They concluded that the approach that treats text as an ordered hierarchy of content objects (OHCO) prevails over other approaches because of its capability to support data integrity, information retrieval functions, and special processing on nontextual data (DeRose et al. 1990). OHCO modeling is regarded as an eligible approach to model JHA documents and is adopted in this research due to the advantages outlined in DeRose et al. (1990). The OHCO approach views the texts of a document as an ordered hierarchy of content objects, which can be described by defining its constituent terms separately and explaining their potential role in this research:

- Content objects represent structural components in a document. For example, a JHA document could have content objects represented as activity objects, job step objects, and hazard objects.

- Hierarchy indicates the content objects are organized into a system with different hierarchical levels. For instance, one activity object may comprise multiple job step objects, and one job step object may be associated with multiple hazard objects in a JHA document.

- Ordered states the arrangement of content objects, i.e., which objects may follow or precede another. For instance, an activity object may also contain one applicability condition object for each job step object, which specifies the condition in which that job step object applies. The applicability condition object shall precede its related job step object to let document readers know at once to what condition the following job step object applies. The content objects of the OHCO approach can be best represented through descriptive markup by putting markup tags around them. For example, the notion that an activity named "frame columns" has four substeps "fly forms to area to be installed," "take forms off cart/blocking," "stand forms into place," and "set pins" may be represented in the OHCO approach as follows:

$$
<\text { Activity }>
$$

$<$ ActName $>$ Frame columns $</$ ActName $>$

$<$ JobStep $>$

$<$ JobStepTitle>Fly forms to area to be installed $</$ JobStepTitle $>$

$</$ JobStep $>$

$<$ JobStep $>$

$<$ JobStepTitle $>$ Take forms off cart/blocking $</$ JobStepTitle $>$

$</$ JobStep $>$

$<$ JobStep $>$

$<$ JobStepTitle $>$ Stand forms into place $</$ JobStepTitle $>$ $</$ JobStep $>$

$<$ JobStep $>$

$<$ JobStepTitle $>$ Set pins $</$ JobStepTitle $>$

$</$ JobStep $>$

$</$ Activity $>$
The markup tag <Activity>, with its respective closing tag $</$ Activity $>$, is the root tag representing the activity content object, in which the activity name and job step content objects are defined through markup tags.

\section{Extensible Markup Language}

To model documents in OHCO through a descriptive markup approach, it is necessary to have a standard that defines the required descriptive markup tags and specifies the rules of using the defined descriptive markup tags to model the content objects of the documents. XML is a widely known, common standard that can serve this purpose and was chosen in this research.

The standard XML became a recommendation of the World Wide Web Consortium (W3C) in 1998 (Harold and Means 2004). $\mathrm{XML}$ is a meta-language that provides a means of defining markup tags to describe content objects of a document. Because XML tags are human-readable texts and the entire modeled document thus is in a human-readable text format, users can easily revise the document models as long as they abide by the rules specified by the defined markup language.

To model documents using XML, an XML schema language must be used to create an XML schema that defines the constituent elements of the XML document and specifies the structural rules for these elements. XML schema definition (XSD), which was developed by W3C (2004b), is adopted in this research because it has better control over the data types and format of elements than other languages (Harold and Means 2004).

\section{Ontology and Ontological Modeling}

Gruber (1993) defined an ontology as "an explicit and formal specification of a conceptualization." An ontology can model a set of concepts and relationships among these concepts within a knowledge domain. Ontological modeling, therefore, is a process to model concepts and relationships into ontologies.

Ontological modeling is a well-suited approach to model JHA concepts for two main reasons. First, JHA concepts and their semantic relationships can be easily represented in the form of classes and properties in an ontology. Second, the applicability of a JHA concept used to describe a specific JHA situation can imply applicability or inapplicability of other JHA concepts that are semantically related to the first concept.

Many research efforts in the computer science domain, such as Wang et al. (2004) and Kim and Choi (2006), have adopted ontological modeling for modeling and reasoning about contextual concepts, which are concepts describing contextual information. Researchers proposed ontology-based frameworks for modeling and reasoning about contextual concepts in pervasive computing environments. In the architecture/engineering/construction and facilities management domain, the number of research works applying ontological modeling to model and reason about contextual concepts is growing. The e-COGNOS project was the first project to deploy a domain ontology for knowledge management and contextual concept modeling and reasoning in the construction industry (Lima et al. 2005). Aziz et al. (2005) used an ontology to represent and deliver contextual information, such as location, time, and profile. Dolenc et al. (2007) developed an ontology framework for modeling contextual concepts in the InteliGrid project.

\section{Web Ontology Language}

To develop a concept ontology, an ontology language is required to provide formal syntactic structure and modeling rules with which concepts can be modeled. Ontology languages allow users to conceptualize and formalize domain knowledge (El-Diraby et al. 2005; Lima et al. 2005; Rezgui 2006). 
The most common ontology languages include resource description framework (RDF) and RDF schema (RDFS), DAML +OIL (DARPA Agent Markup Language+Ontology Inference Layer), and web ontology language. OWL is a specification by W3C (2004a) and serves as a fundamental component of the Semantic Web initiative (Antoniou and van Harmelen 2004). OWL is based on the DAML+OIL language and therefore has many features of DAML+OIL, such as adopting RDF as the modeling language to define ontology vocabularies and using XML-based RDF syntax for representing information (Bechhofer et al. 2004). OWL is an extension of RDFS in the sense that OWL uses the RDF meaning of classes and properties (rdfs:Class, rdfs:subClassOf, and other rdfs syntax) and adds language primitives to support a richer expressiveness. Chen et al. (2003) argued that, as a result, OWL has better expressiveness than other ontology languages. Therefore, the proposed framework adopts OWL to exploit and build upon this expressiveness.

\section{Proposed Framework}

The proposed framework includes two parts: the representation model and reasoning mechanism. The representation model aims to provide a systematic structure for modeling JHA knowledge in a computer-interpretable format. The representation model represents each safety rules' corresponding JHA concepts in a concept ontology and also represents JHA documents in XML format. The reasoning mechanism allows evaluating JHA concepts and XML-based JHA documents stored in the representation model to determine each safety rule's applicability. In the following section, the representation model is discussed first, followed by the discussion of the reasoning mechanism.

\section{Representation Model}

The representation model consists of two submodels: the concept representation model and document representation model. The former aims to model JHA concepts in an ontology, and the latter aims to model JHA documents in XML format. The two submodels are discussed in the following sections.

\section{Concept Representation Model}

The concept representation model leverages ontological modeling to model JHA concepts extracted from JHA documents and to model relationships among these concepts. When tied to JHA documents, JHA concepts are used to describe the applicability conditions of safety rules. As input to the reasoning mechanism, the concepts are selected to represent a list of potential situations that may occur in a project, i.e., the project-specific contexts. Hence, JHA concepts can be viewed as a set of topological concepts of a JHA domain knowledge base, and these topological concepts and the relationships among them should be organized to benefit the evaluation process for identifying the applicability of JHA safety rules.

In this research, OWL is used to model JHA concepts in a concept ontology, and Protégé, an open-source ontology editor (Protégé 2008), is leveraged to implement the ontological modeling process. A guide to developing OWL-based ontologies using Protégé can be found in Horridge et al. (2009) and is out of the scope of this paper.

Although different approaches to develop ontologies have been discussed (Darlington and Culley 2008), every approach has its own purpose depending on its application. An approach suitable for one application domain may not be suitable for another (Breitman et al. 2006). Therefore, this paper proposes the following steps to build a concept ontology, which take necessary considerations of the concept representation model into account. The steps include
(1) selecting classifications and categorizing concepts, and (2) defining relationships and connecting concepts.

Step 1: Selecting classifications and categorizing concepts. The first step is to define classifications for structuring JHA concepts in the concept ontology. In the classifications, three primary classes, activity, job step, and hazard, are defined for the three types of JHA concepts (i.e., activities, job steps, and hazards). In addition to these three primary classes, it was noted previously that the three types of JHA concepts have respective semantic properties, which affect how concepts of each type can be further categorized in the primary classes. To consider these semantic properties, the following strategies are adopted in this research to develop secondary classes for each primary class:

- The classification of MasterFormat (Construction Specifications Institute 2005) is used as secondary classes under the primary activity class to further represent activity concepts in the concept ontology. MasterFormat provides a detailed classification system for assigning construction specifications to divisions. Thus, MasterFormat is well-suited for activity concepts to represent their division-specific semantic properties.

- A classification that comprises those action concepts appearing in the job step concepts is used as the secondary classification under the primary job step class to represent job step concepts in the concept ontology. This is because job step concepts can be most straightforwardly grouped based on the action concepts specified in the job step concepts' titles since each job step concept carries at least one action concept. For job step concepts comprising multiple action concepts, only the most representative action concept of the job step concept is selected and incorporated in the set of secondary classes.

- A classification adapted from the Occupational Injury and Illness Classification Manual (OI\&ICM) (U.S. Department of Labor 1992) is used as the secondary classification under the primary hazard class to represent hazard concepts in the concept ontology. Hazard concepts are more diversified than the other two types of concepts. Therefore, a well-organized classification is especially necessary for the representation of and reasoning about hazard concepts. The OI\&ICM classifies potential hazards according to their exposure. The classification that is adapted from OI\&ICM and used in this research includes the following classes: contact with objects and equipment, falls, bodily reaction and exertion, exposure to harmful substances or environments, transportation accidents, fires and explosions, and other events or exposures. These classes provide an organized structure that is well-suited for representing diversified hazard concepts in this research.

In addition, other intermediate classifications for further classifying concepts under the secondary classes may be necessary when JHA concepts to be represented in nature are subdivisions of the intermediate classifications. For example, the seven secondary classes adapted from OI\&ICM have their respective subclasses. If their subclasses are also suitable to further classify hazard concepts, these subclasses should be incorporated as the tertiary classes in the concept ontology.

The primary, secondary, and other incorporated intermediate classifications form a hierarchical structure in the concept ontology. Each JHA concept can be categorized into one of the classification's classes as a subclass. For example, the activity concept "frame columns" can be categorized as a subclass of the secondary class concrete activity, which is a subclass of the primary activity class.

Step 2: Defining semantic relationships and connecting concepts through these relationships. When JHA concepts are represented in classifications, superclass-subclass relationships 
between the concepts are specified. Additional semantic relationships, such as associations, also need to be specified between the concepts to represent the semantic connections between the concepts and to benefit later reasoning about the concepts by enabling navigation between related concepts.

Associations are semantic connections between concepts that do not have superclass-subclass relationships between them and specify the relatedness, exclusiveness, or identicalness of two concepts' meaning. Two main types of associations are used in this research: nonlogical associations (called association relationships) and logical associations (called logical relationships).

When defining association relationships to connect concepts, the association relationships should be given semantically rich names to facilitate the understanding of how connected concepts are related (Wang and Boukamp 2008). In this research, the primary activity class has an association relationship has Step connecting the primary job step class in representing the semantic connection of "an activity hasStep job step(s)". Similarly, another association relationship hasHazard is defined to enable the semantic connection of the primary job step and hazard classes. These two association relationships are then defined in the concept ontology in the form of relationship properties for the primary classes.

Logical relationships include disjoint and equivalent relationships, which specify the exclusiveness and identicalness of concepts, respectively. Disjoint relationships connect concepts that exclude one another. For example, the two concepts "fall from rolling scaffold" and "fall from suspended scaffold" are declared disjoint because someone falling from one type of scaffold usually cannot fall from another type of scaffold at the same time. On the other hand, equivalent relationships connect concepts that have the same semantic meaning. For instance, a hazard concept "slip" can be declared equivalent to another hazard concept "trip" because they both mean that someone accidently slides or falls and loses his/her balance in the context of construction safety. The safety implications therefore are the same for both concepts.

Association relationships and logical relationships are important to help semantically string related JHA concepts together, which enables propagation of applicability values among concepts for effective knowledge inference from given facts about JHA concepts' applicability. The interpretation of all the relationships for the purpose of ontological reasoning is discussed in detail in a subsequent section.

\section{Document Representation Model}

The document representation model leverages XML to represent JHA documents in a computer-interpretable format. To use the document representation model to represent a JHA document in XML format, the steps to be taken consist of (1) defining an XML schema, and (2) representing JHA documents in XML format according to the specification of the schema.
Step 1: Defining an XML schema. The first step of the document representation is to define an XML schema that formalizes the structure of JHA documents. The purpose of defining the schema is to specify what markup tags should be defined to represent the elements and how they are organized in the XML representation. Only the basic notions of how to use XSD to define the schema are discussed in the following paragraphs; the details of XSD are out of the scope of this research and can be found in W3C (2004b).

First, the XSD element <xs:element> is used to define a new XML tag named JHA as the root tag for a JHA document and assign a user-defined data type "JHAType" to the JHA tag. A userdefined data type assigned to a tag specifies what other tags will be nested in that tag. For example, "JHAType" is defined, through $<x s$ :complexType $>$, as a data type that can in sequence present multiple activity tags. In other words, this definition specifies that a JHA tag can contain multiple activity tags in itself. These are illustrated in Fig. 2, a snippet of the XSD for JHA documents.

Because the activity tag is assigned another user-defined data type "ActType," the rest of the snippet in Fig. 2 continues defining this new data type. The schema declares that "ActType" is a data type that can in sequence present five tags: ActTitle tag, which surrounds the activity's name; Ctxt_App_Condition tag, which surrounds the concepts representing the applicability conditions of JHA knowledge; ActDescription tag, which surrounds the activity's description; and JobStep tag, which groups the job step information of the activity.

In summary, following the schema definition process, the JHA documents' four constituent elements are formalized by giving them respective tags, such as <Activity/> for the activity element. In addition, three other types of tags are also defined in the schema in this research.

- Descriptive and informative tags: These tags are defined to further elaborate the four constituent elements, such as $<$ ActTitle/ $>$ and $<$ ActDescription/ $>$ for elaborating activity title and description. For example, the activity in the JHA document in Fig. 1 can be represented in XML format as

$<$ ActTitle $>$ Frame Columns $</$ ActTitle $>$

$<$ ActDescription $>$ This job is about using formwork to frame columns $<$ /ActDescription $>$

- Concept tags: These tags are <Concept/>, <Concept_AND/>, and $<$ Concept_OR/ $>$. They are defined to indicate the information of concepts and are only used in the reasoning tags, discussed next, to specify applicability conditions of JHA knowledge. If an applicability condition of JHA knowledge is described through a single concept, the tag <Concept/> should be used, such as " $<$ Concept $>$ Frame Column $</$ Concept $>$." If an applicability condition of JHA knowledge is described through multiple concepts, these concepts have to be respectively surrounded with the tag $<$ Concept/ $>$; $<$ Concept_AND/ $>$ or $<$ Concept_OR/ $>$ is then used

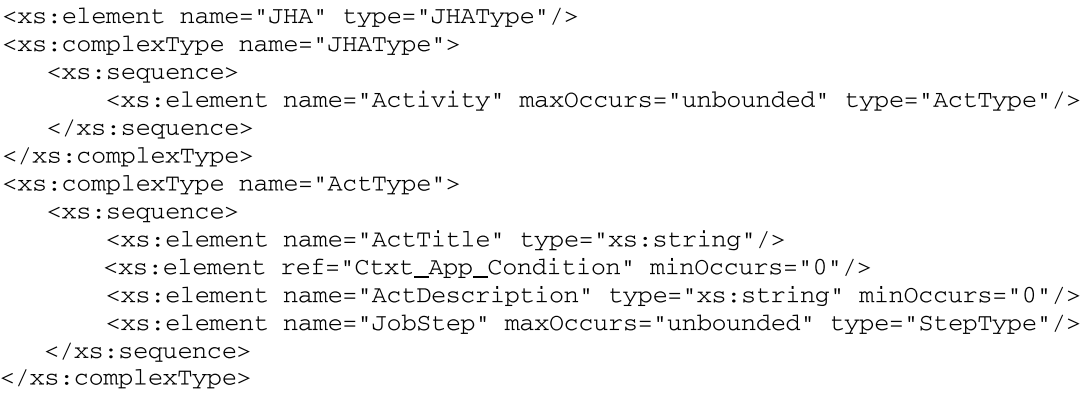

Fig. 2. Snippet of an XSD for the example JHA document 
to surround all the concepts. For example, JHA knowledge

"frame and pour columns" (described through the concept conjunction "frame columns AND pour columns") is represented in XML as

\section{$<$ Concept_AND $>$}

$<$ Concept $>$ Frame Columns $</$ Concept $>$

$<$ Concept $>$ Pour Columns $</$ Concept $>$

$</$ Concept_AND $>$

Whether $<$ Concept_AND/ $>$ or $<$ Concept_OR/ $>$ should be used for multiple concepts depends on whether the multiple concepts are conjunctively or disjunctively related.

- Reasoning tag: This tag is <Ctxt_App_Condition/>, which is defined to describe the applicability conditions of JHA knowledge. This tag will contain exactly one of the concept tags because applicability conditions are described through either a concept or a concept conjunction or a concept disjunction.
For example, to represent JHA knowledge "frame and pour columns" as an applicability condition, this condition is represented in XML as

$<$ Ctxt_App_Condition $>$

$<$ Concept_AND $>$ $<$ Concept $>$ Frame Columns $</$ Concept $>$ $<$ Concept $>$ Pour Columns $</$ Concept $>$ $</$ Concept_AND $>$

\section{$</$ Ctxt_App_Condition $>$}

All the tags that are defined in the schema for representing JHA documents are summarized in Table 1. Table 1 also shows which tags are necessary in an XML document, which are optional, and how tags should be nested.

Step 2: Representing JHA documents in XML format. The second step of the document representation model is to represent JHA documents in XML format using the defined tags listed in

Table 1. Listing of the Defined XML Markup Tags for JHA Documents

\begin{tabular}{|c|c|c|c|c|c|}
\hline Number & Markup name & Markup symbol & Markup description & Inner element ${ }^{\mathrm{a}}$ & Optional \\
\hline 1 & JHA & $<\mathrm{JHA} />$ & Root tag, starting and ending a JHA document & 1 & No \\
\hline 2 & Activity & $<$ Activity/> & Tag grouping activity information & $3,4,5,13$ & No \\
\hline 3 & Activity title & $<$ ActTitle/> & Tag surrounding the activity's title & String & No \\
\hline 4 & Activity description & $<$ ActDescription/> & Tag surrounding the activity's description & String & Yes \\
\hline 5 & Job step & $<$ JobStep $>>$ & Tag grouping job step information & $6,7,8,13$ & No \\
\hline 6 & Job step title & $<$ StepTitle/> & Tag surrounding the job step's title & String & No \\
\hline 7 & Job step description & $<$ StepDescription/> & Tag surrounding the Job Step's description & String & Yes \\
\hline 8 & Hazard & $<$ Hazard/> & Tag grouping hazard information & $9,10,11,13$ & No \\
\hline 9 & Hazard title & $<$ HazardTitle/> & Tag surrounding the Hazard's title & String & No \\
\hline 10 & Hazard description & $<$ HazardDescription/> & Tag surrounding hazard's description & String & Yes \\
\hline 11 & $\begin{array}{l}\text { Recommended } \\
\text { safety procedure }\end{array}$ & $<$ RecommendedProcedure/ $>$ & Tag grouping recommended procedure information & 12 & No \\
\hline 12 & Rule & $<$ Rule/ $>$ & Tag surrounding the rule for the recommended procedure & String & No \\
\hline 13 & Applicability condition & $<$ Ctxt_App_Condition/> & $\begin{array}{l}\text { Tag grouping the applicable conditions of } \\
\text { the safety requirements }\end{array}$ & 14 or 15 or 16 & No \\
\hline 14 & Concept & $<$ Concept $/>$ & Tag surrounding exactly one concept & String & Yes \\
\hline 15 & Concept conjunction & $<$ Concept_AND/> & Tag representing conjunction of multiple concepts & 14,16 & Yes \\
\hline 16 & Concept disjunction & $<$ Concept_OR/> & Tag representing disjunction of multiple concepts & 14,17 & Yes \\
\hline
\end{tabular}

${ }^{\mathrm{a}}$ The content inside a tag, which is a tag or a set of tags or a string.

Table 2. Test Case Results of Assigning Applicable Value to Multiple Concepts

\begin{tabular}{|c|c|c|c|c|c|c|c|c|}
\hline \multirow[b]{2}{*}{$\begin{array}{l}\text { Test } \\
\text { case }\end{array}$} & \multirow[b]{2}{*}{ Test concept } & \multirow[b]{2}{*}{$\begin{array}{l}\text { Assigned } \\
\text { applicability } \\
\text { value }\end{array}$} & \multicolumn{2}{|c|}{ Separate evaluation } & \multicolumn{4}{|c|}{ Combined evaluation } \\
\hline & & & $\begin{array}{l}\text { Number of } \\
\text { applicable } \\
\text { safety rules }\end{array}$ & $\begin{array}{l}\text { Number of } \\
\text { not- } \\
\text { applicable } \\
\text { safety rules }\end{array}$ & $\begin{array}{l}\text { Number of } \\
\text { applicable } \\
\text { safety rules }\end{array}$ & $\begin{array}{l}\text { Number of not } \\
\text { applicable } \\
\text { safety rules }\end{array}$ & $\begin{array}{l}\text { Applicable } \\
\text { safety rules } \\
\text { identified }\end{array}$ & $\begin{array}{l}\text { Not- } \\
\text { applicable } \\
\text { safety rules } \\
\text { identified }\end{array}$ \\
\hline \multirow[t]{2}{*}{1} & Frame columns & & 20 & 0 & & & & \\
\hline & $\begin{array}{l}\text { Excavation using } \\
\text { support systems }\end{array}$ & Applicable & 29 & 23 & 49 & 23 & True & True \\
\hline \multirow[t]{2}{*}{2} & Forklift use & & 11 & 7 & & & & \\
\hline & $\begin{array}{c}\text { Decontamination } \\
\text { of windows }\end{array}$ & Applicable & 15 & 9 & 26 & 1 & True & True \\
\hline \multirow[t]{2}{*}{3} & Carcinogen control & \multirow{2}{*}{ Not applicable } & 0 & 13 & \multirow{2}{*}{0} & \multirow{2}{*}{27} & \multirow{2}{*}{$\mathrm{n} / \mathrm{a}$} & \multirow{2}{*}{ True } \\
\hline & Spray painting & & 0 & 14 & & & & \\
\hline \multirow[t]{2}{*}{4} & Frame columns & Applicable & 20 & 0 & \multirow{2}{*}{15} & \multirow{2}{*}{5} & \multirow[t]{2}{*}{ True } & \multirow[t]{2}{*}{ True } \\
\hline & Set pins ${ }^{\mathrm{a}}$ & Not applicable & 0 & 5 & & & & \\
\hline
\end{tabular}

aThis is a job step concept of the activity concept "Frame Columns." 
Table 2 and following the schema definition. Fig. 3 illustrates a snippet of an XML document that is based on the JHA document in Fig. 1

As noted previously, applicability conditions of JHA knowledge are described through concepts representing activities, job steps, and hazards. Because of the hierarchical structure of JHA knowledge in JHA documents, the descriptions of applicability conditions of JHA knowledge shall expand with the development of the hierarchical structure from the tag activity to the tag hazard in a document. Specifically, the applicability condition of an activity can be sufficiently described through a single activity concept, and that of a hazard or recommended safety procedure should be described through activity, job step, and hazard concepts jointly. For example, as shown in Fig. 3, only one concept "frame columns" is needed in the hierarchical level of $<$ Activity/>, but another concept "fly forms to area to be installed" is added into the applicability condition and made conjunctive to the first concept in the hierarchical level of $<$ JobStep/>. This characteristic of applicability condition expansion takes into account different applicability conditions that each hierarchical level introduces.

\section{Reasoning Mechanism}

The reasoning mechanism consists of two submechanisms: the concept evaluation mechanism and document evaluation mechanism. The concept evaluation mechanism aims to evaluate the applicability of each JHA concept of the concept ontology according to a set of concepts for which the applicability values are known. The document evaluation mechanism aims to evaluate the applicability of JHA knowledge represented in XML format and to classify evaluated JHA knowledge based on their applicability. The two submechanisms are discussed in the following sections.

\section{Concept Evaluation Mechanism}

The concept evaluation mechanism leverages the relationships defined in the concept ontology to evaluate each concept's applicability, specifically propagating applicability values throughout the concept ontology. Each JHA concept of the concept ontology has three possible applicability values: applicable, not applicable, and possibly applicable. Possibly applicable is the initial applicability value for concepts. Each concept must carry exactly one of these applicability values at any given time. The values applicable and not applicable mean the situation described by the concept carrying the value applies and does not apply to the current situation, respectively. If a concept is possibly applicable, it means the information to determine whether the concept applies or does not apply to a situation is insufficient; therefore, the applicability is unknown. For example, if an engineer observes that a column-framing activity is underway on site, the concept "frame columns" can be flagged applicable. If the engineer knows for sure that no column-framing activity occurs, the concept can be flagged not applicable. Otherwise, the concept remains possibly applicable, and its applicability value can be updated when further information about the activity is available.

Concept evaluation principles. The concept evaluation mechanism requires as input known applicability values for some JHA concepts. Specifically, these concepts carry either the value applicable or not applicable. During the evaluation process these applicability values are then propagated to other JHA concepts which are connected to these concepts through the defined relationships. The propagation thereby follows these four concept evaluation principles.

Principle 1: If a JHA concept is applicable, its superconcepts must be applicable. If a JHA concept is not applicable, its subconcepts must be not applicable. A JHA concept's superconcepts are the generalization of the concept. If the JHA concept is applicable, its generalized superconcepts must have the same applicability. Similarly, a JHA concept's subconcepts are the specialization of the concept. If the JHA concept is not applicable, its specialized subconcepts must be not applicable.

Principle 2: If a JHA concept is applicable, another JHA concept that is connected to the first concept through an association relationship must be applicable. This principle only applies when a JHA concept is found applicable. The association relationships are used to semantically connect JHA concepts that are related with

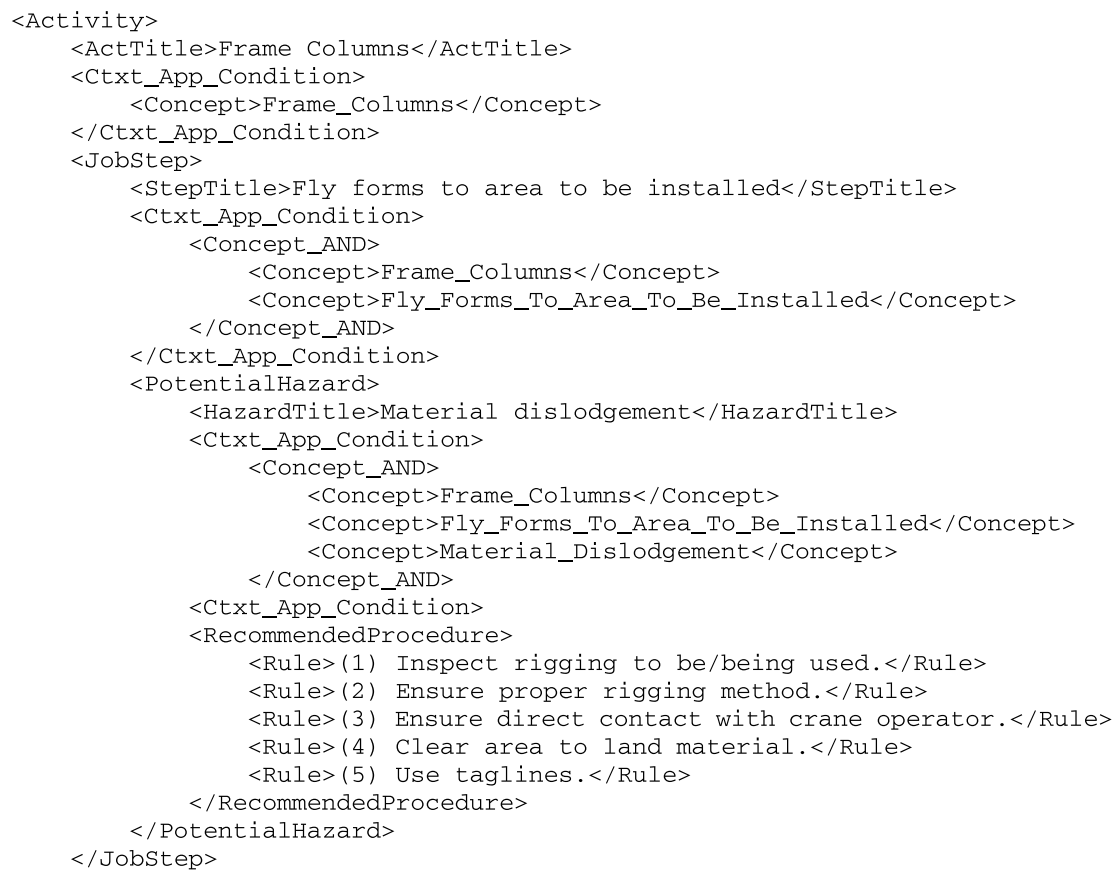

Fig. 3. Snippet of the example JHA document in XML format 
each other and, therefore, indicate that when the connecting concept is applicable, the connected concept should also be applicable. For example, the job step concept "set pins" connects with the hazard concept "fall" through an association relationship hasHazard. Once the concept "set pins" is found applicable, the concept "fall" will accordingly become applicable.

Principle 3: Two JHA concepts connected through an equivalent association relationship must carry the same applicability value. Two JHA concepts that are connected through an equivalent association relationship have the same contextual meaning, and therefore, must share the same applicability value, no matter whether this value is applicable, not applicable, or possibly applicable.

Principle 4: If a JHA concept is applicable, another JHA concept that is connected to the first JHA concept through a disjoint association relationship must be not applicable. This principle only applies when a JHA concept is found applicable. JHA concepts that are connected through disjoint association relationships semantically exclude one another. Therefore, when one concept is applicable, this concept will exclude other concepts to which it connects through disjoint relationships and require that those concepts be not applicable.

These four principles guide the evaluation process when the evaluation mechanism is activated. After the evaluation process, the concept ontology is updated, and some JHA concepts in the concept ontology will carry an applicability value different from the initial value of possibly applicable. These are the JHA concepts that are assigned different values in the beginning to describe a certain project situation and the JHA concepts to which different applicability values are propagated following these evaluation principles. During the evaluation process, it is possible to encounter applicability contradictions for a concept, e.g., a concept may first be found applicable but then later assigned the contradicting value not applicable. The following discusses when and how concept applicability contradictions may occur and how a contradiction can be resolved.

Concept applicability contradiction. This research observes two causes for concept applicability contradiction. They are (1) a flawed concept ontology, and (2) application of the reasoning principles to multiple input concepts.

A flawed concept ontology. If required relationships are not defined between concepts or not appropriately used to connect concepts, the concept ontology may represent the domain knowledge incorrectly, which can result in applicability contradictions when the evaluation mechanism interprets the concept ontology. For example, if two concepts, each of which has a superconcept, are connected through an association relationship and their superconcepts are declared disjoint with each other, applicability contradiction will occur. The reason is when two concepts are associated, it implies that their respective superconcepts also are associated in a way and therefore, should not be disjoint.

Application of the reasoning principles to multiple input concepts. If the concept ontology is not flawed, an applicability contradiction may still occur as a result of applying the reasoning principles to multiple input concepts. For example, suppose that two disjoint concepts both apply to a project situation. When one concept is first specified applicable in the evaluation process, the other will be automatically evaluated to be not applicable. However, when the other concept is provided as an additional input with the value applicable, it contradicts the applicability value determined by the reasoning mechanism.

To address applicability contradictions, a contradiction detection mechanism was implemented in the evaluation mechanism. This contradiction detection mechanism is able not only to send warning messages to users when a contradiction is detected, but also to provide users with options to accept or decline the new, contradicting applicability values of concepts. When warning messages are sent to users, e.g., in the form of a pop-up dialogue on the screen, users should first respond to the warning messages by determining to accept or decline the new applicability value that results in the contradiction. At this point, users get a chance to evaluate the alternatives of the applicability determined by the evaluation mechanism and the one obtained from the initial input and determine which applicability value better describes the current project situation. For instance, in the second example discussed previously, one should accept the suggested applicability value, i.e., applicable, which conflicts with the evaluated value, i.e., not applicable, for the second concept as it applies to the project situation.

On the other hand, users or ontology developers need to inspect the concept ontology to see whether there is any flaw that results in a contradiction in the evaluation process. If applicable, the concept ontology should be corrected.

\section{Document Evaluation Mechanism}

The goal of the document evaluation mechanism is to evaluate the applicability of JHA knowledge, specifically JHA safety rules, that is represented in XML format and to classify evaluated JHA knowledge/safety rules based on their applicabilities. To evaluate the applicability of JHA knowledge actually is to evaluate the applicability conditions of the JHA knowledge represented in XML documents. In addition, because applicability conditions are described through JHA concepts, the document evaluation mechanism relies on the availability of applicability values for the JHA concepts. The applicability values are then evaluated to determine whether those applicability conditions are satisfied.

Five evaluation rules are defined in the document evaluation mechanism for determining the applicability of JHA knowledge. The first four rules aim at evaluating the satisfaction value of applicability conditions. The three satisfaction values satisfied, possibly satisfied, and not satisfied are defined as possible evaluation results for applicability conditions. The last rule aims at using these evaluation results to determine JHA knowledge's applicability.

In the following section, first the rules for evaluating applicability conditions are discussed. This is followed by the discussion on the rules for determining JHA knowledge's applicability.

Rules for evaluating applicability conditions. JHA knowledge in any hierarchical level has exactly one applicability condition, which must be evaluated according to the rules prescribed in this section. To facilitate the understanding of the rules, the term "evaluation unit" is adopted in the rules. An evaluation unit is defined as a part of an applicability condition to be evaluated to determine whether the condition is satisfied or not. The following applies to evaluation units:

- An evaluation unit can be a single concept, a concept conjunction, or a concept disjunction. In case of a concept conjunction or disjunction, the evaluation unit has subevaluation units.

- To evaluate an evaluation unit, its subevaluation units should be evaluated first.

- An evaluation unit has exactly one of three applicability values: applicable, possibly applicable, and not applicable.

- An applicability condition can have only one root evaluation unit, meaning it is the outermost evaluation unit in an applicability condition. A root evaluation unit may have subevaluation units.

Fig. 4 shows an example applicability condition for a JHA document, in which the root evaluation unit consists of two subevaluation units; one of the two subevaluation units further contains two other subevaluation units. 


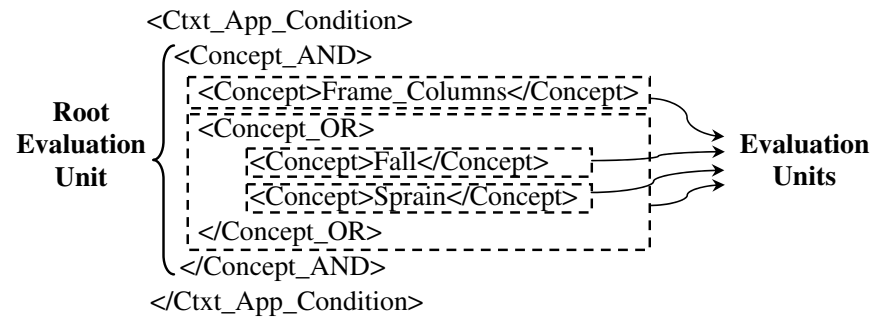

Fig. 4. Example applicability condition demonstrating root and subevaluation unit

The first evaluation rule defines the relation between applicability conditions and evaluation units.

Rule 1: Applicability condition evaluation rule. The satisfaction values of applicability conditions are determined by the applicability values of their root evaluation units. If the root evaluation unit is applicable, possibly applicable, or not applicable, the conditions will be satisfied, possibly satisfied, or not satisfied, respectively.

The following rules are applied to evaluate the root evaluation unit and its subevaluation units. The applicability condition shown in Fig. 4 is used as an example to demonstrate the following rules.

Rule 2: Concept evaluation rule. The applicability value of an evaluation unit that is a single concept must be the same as the applicability value of the single concept. For example, if the concept "fall" is applicable, the evaluation unit $<$ Concept $>$ Fall $</$ Concept $>$ shown in Fig. 4 is also applicable.

Rule 3: Concept disjunction evaluation rule. The applicability value of an evaluation unit that is a concept disjunction can be determined as follows:

a. The evaluation unit must be applicable if at least one of its subevaluation units is found applicable. For example, if the subevaluation unit $<$ Concept $>$ Fall $</$ Concept $>$ of the concept disjunction in Fig. 4 is found applicable, the concept disjunction will be applicable, no matter whether the other subevaluation unit of the concept disjunction is applicable or not.

b. If at least one of the subevaluation units is possibly applicable and none of the others is applicable, the evaluation unit must be possibly applicable. For example, if the subevaluation unit $<$ Concept $>$ Fall $<$ /Concept $>$ of the concept disjunction in Fig. 4 is possibly applicable and the other subevaluation unit $<$ Concept $>$ Sprain $</$ Concept $>$ is not applicable, the concept disjunction will be possibly applicable.

c. Otherwise, if all the subevaluation units are not applicable, the evaluation unit must be not applicable. For example, if both the subevaluation units in Fig. 4 are not applicable, the concept disjunction will be not applicable.

Rule 4: Concept conjunction evaluation rule. The applicability value of an evaluation unit that is a concept conjunction can be determined as follows:

a. An evaluation unit must be not applicable if at least one of its subevaluation units is found not applicable. For example, if the subevaluation unit $<$ Concept $>$ Frame_Columns $</$ Concept $>$ of the concept conjunction in Fig. 4 is found not applicable, the concept conjunction will be not applicable, no matter whether the other concept conjunction's subevaluation unit, which is a concept disjunction surrounded by $<$ Concept_OR $>$ $</$ Concept_OR $>$, is applicable or not.

b. If at least one of the subevaluation units is possibly applicable and all the others are applicable, the evaluation unit must be possibly applicable. For example, if the subevaluation unit $<$ Concept $>$ Frame_Columns $</$ Concept $>$ of the concept conjunction in Fig. 4 is possibly applicable and the other subevaluation unit is applicable, the concept conjunction will be possibly applicable.

c. Otherwise, if all the subevaluation units are applicable, the evaluation unit must be applicable. For example, if the subevaluation units $<$ Concept $>$ Frame_Columns $</$ Concept $>$ and the concept disjunction in Fig. 4 are applicable, the concept conjunction is applicable.

Rules for determining JHA knowledge's applicability. After applicability conditions are evaluated using the aforementioned evaluation rules, the applicability of the JHA knowledge can be evaluated using the following rules.

Rule 5: JHA knowledge evaluation rule. JHA knowledge in a hierarchical level is applicable, not applicable, or possibly applicable only when the applicability condition for the JHA knowledge is satisfied, not satisfied, or possibly satisfied, respectively.

These five evaluation rules are defined for the document evaluation mechanism. The evaluation process starts with evaluating applicability conditions by deploying the Rules 1 to 4 . Then the evaluation process applies the Rule 5 to determine the applicability of JHA knowledge based on the satisfaction values of the applicability conditions. For example, if the applicability condition of JHA knowledge in a hierarchical level is satisfied, the JHA knowledge is applicable. Once JHA knowledge's applicability is obtained, it can be classified according to their applicability, and the applicable JHA safety rules that apply to the project's situations become available.

Concept neglect mechanism. Another important mechanism that was developed in the document evaluation mechanism is concept neglect, which means to neglect certain concepts describing applicability conditions in an evaluation process such that these concepts' applicability values are ignored and not evaluated by the evaluation mechanism.

JHA safety rules are tied to a combination of activity, job step, and hazard concepts, and they also can be tied to hazard concepts alone. Engineers may want to know what JHA safety rules are applicable when certain job steps are performed, regardless of the hazards associated with the job steps, or when certain hazards occur on sites no matter what steps are taken. Such a search can be realized in this research by implementing the concept neglect mechanism in the document evaluation mechanism. Fig. 5 is an example of how concept neglect works. If the activity concept "frame columns" is neglected during the evaluation process, then the evaluation mechanism will only evaluate the concept "fall." In this case, the original applicability condition with the concept conjunction becomes equivalent to an applicability condition described through a single concept "fall."

The concept neglect mechanism allows engineers to search for JHA knowledge which are associated with specific JHA concepts or specific types of JHA concepts while ignoring others. It enhances the document evaluation mechanism by increasing the mechanism's capability of evaluating JHA knowledge under different user-specified conditions. The evaluation mechanism, including the concept neglect mechanism, are validated and deployed in case studies discussed in the next section.

\section{Case Studies}

To validate the proposed framework, Java programming language (JDK 1.6.0) was used to develop a prototype system, JHA Adviser, which supports the use of the proposed framework. The prototype system was deployed within different test case scenarios, which are based on JHA documents acquired from the construction industry. 


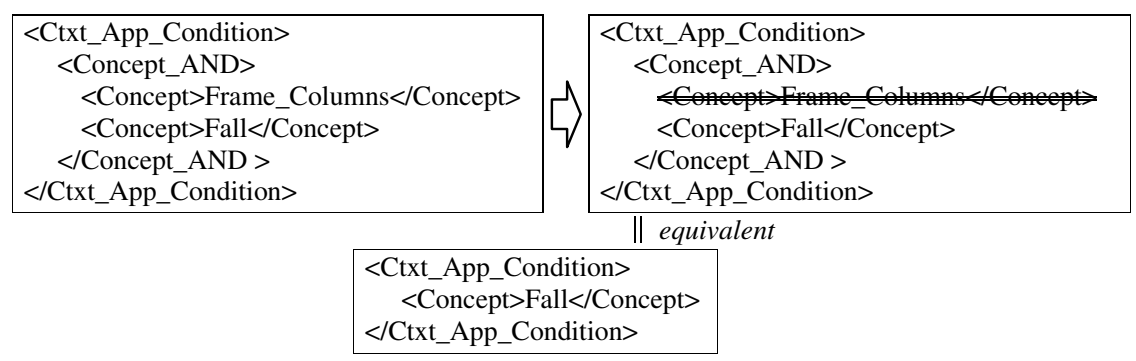

Fig. 5. Illustrative example of concept neglect

The prototype system was used to evaluate the acquired JHA documents and their JHA concepts modeled for these test cases.

In the test cases, there are 78 JHA documents acquired from a private construction company. These documents covered job hazard analyses for different divisions of construction, such as concrete construction, excavation, electrical construction, masonry, mechanical construction, and plumbing. They together accounted for a combined total of 678 JHA concepts and 1,121 JHA safety rules. According to safety professionals' opinions, these documents best represent the document writers' JHA knowledge related to the topics of these documents. Therefore, it is reasonable to assume there is no missing safety knowledge related to these acquired documents' topics and to assume the JHA safety rules in these documents can be used as prior knowledge in the validations. These assumptions ensure that the validations of the aforementioned document evaluation mechanism can focus on these acquired JHA documents without the issues of incomplete safety knowledge related to these documents.

In the following subsections, first the JHA Adviser is briefly discussed, and then the case studies for validating the representation model and reasoning mechanism are reviewed.

\section{Prototype System: JHA Adviser}

JHA Adviser was designed to perform the developed reasoning mechanisms. However, JHA Adviser was not designed to generate the initial input information that JHA Adviser requires, i.e., the initial concept ontology for organizing JHA concepts and relationships in OWL format and JHA documents for storing JHA knowledge in XML format. Therefore, the initial input information must be prepared through other ontology authoring tools, such as Protégé, and document modeling tools, such as Windows Notepad.

Fig. 6 shows the user interface of JHA Adviser. On the left is the working area for the concept ontology, in which three separate panes show the ontology hierarchies of the three primary classes, activity, job step, and hazard. On the right is the working area for JHA documents. Once the concept ontology and JHA documents are prepared in OWL and XML, the following summary steps can be adopted to run JHA Adviser [the detailed steps are illustrated in Wang (2010)]: (1) load the concept ontology into JHA Adviser; (2) load the modeled JHA documents into JHA Adviser; (3) specify applicability values for concepts; (4) propagate applicabilities among the concepts and the JHA documents; and (5) output the

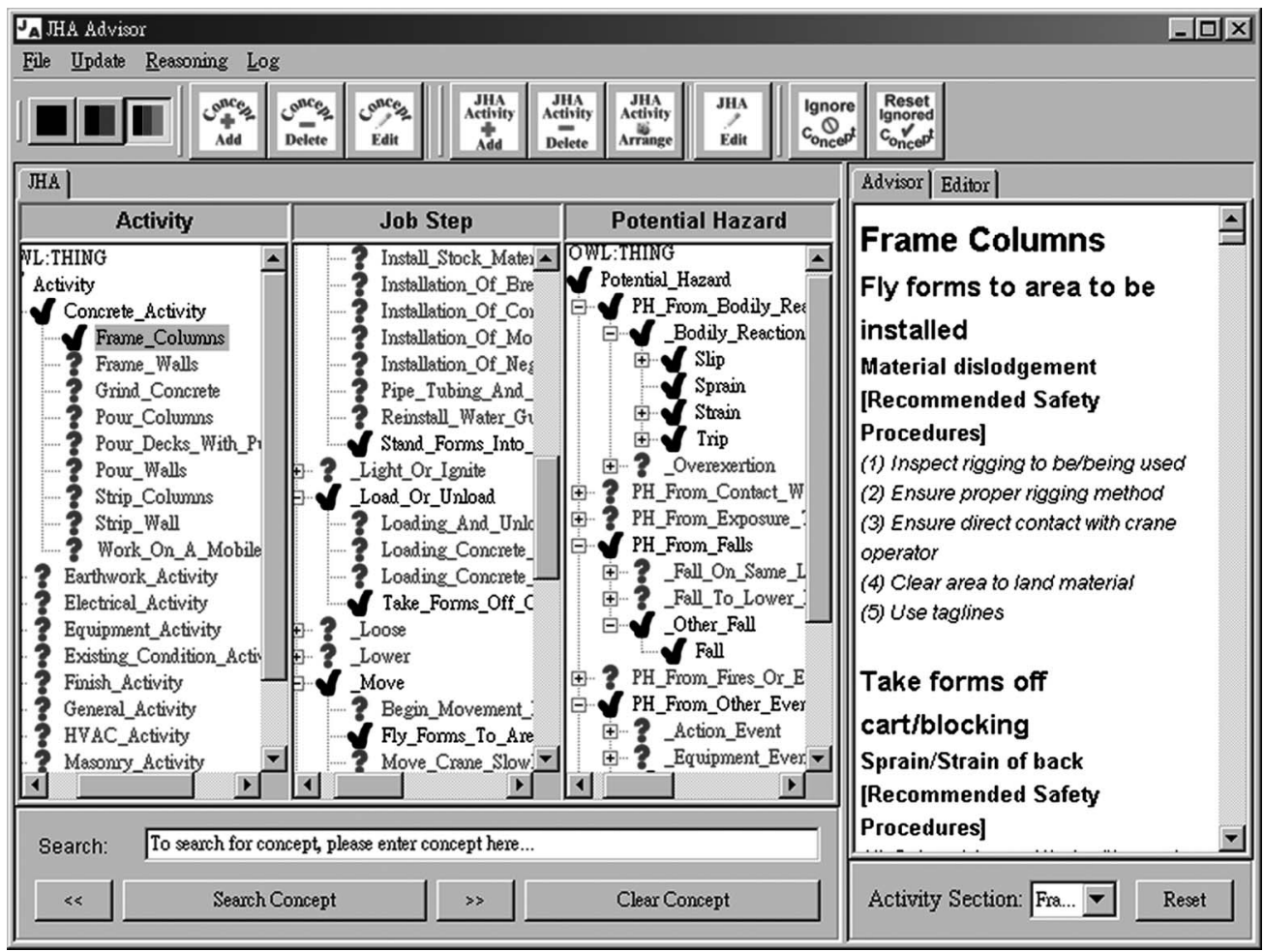

Fig. 6. JHA Advisor's user-interface layout 
reasoning results as a Microsoft Excel spreadsheet file through JHA Adviser.

After the evaluation processes, reasoning results first are shown in JHA Adviser. For JHA concepts, applicable concepts turn green with check marks, possibly applicable concepts remain blue with question marks, and not applicable concepts turn red with cross marks (not shown in Fig. 6). For JHA documents, JHA knowledge is shown using the same coloring scheme. After the reasoning results are output, users can obtain lists of JHA knowledge, categorized according to their applicability, including JHA safety rules together with their related JHA concepts. This process of using JHA Adviser helps perform the aforementioned reasoning mechanism in the case studies for validating the proposed framework.

\section{Case Studies for Validating the Representation Model}

Case studies were performed to validate the effectiveness of using the representation model to represent JHA concepts and JHA documents.

\section{Case Studies for Validating the Concept Representation Model}

The concept representation model was applied to represent the extracted 678 JHA concepts into a concept ontology and test the effectiveness of the representation model. Of the extracted $678 \mathrm{JHA}$ concepts, 78 were activity concepts, 245 were job step concepts, and 355 were hazard concepts. The following summarizes the concept representation process.

- To represent the 78 activity concepts, 10 secondary classes were defined by referring to the classification of MasterFormat (Construction Specifications Institute 2005) under the primary activity class. The 10 secondary classes are concrete activity, excavation activity, electrical activity, equipment activity, existing condition activity, finish activity, general activity, HVAC activity, masonry activity, mechanical activity, plumbing activity, and structural activity. The entire 78 activity concepts can be categorized into these 10 secondary classes. Also, there were no other intermediate classifications used in representing the activity concepts.

- To represent the 245 job step concepts, 43 secondary classes were defined under the primary job step class according to the strategy proposed in the concept representation model. These secondary classes include classes such as adjust, clean, drill, excavate, install, inspect, move, pour, turn off, turn on, and weld. In addition, other intermediate classifications were used in representing these job step concepts. The classifications were formed by some of the job step concepts, which help categorize other concepts. For example, the concept "turn power on" is categorized as a subclass of the turn on class and then also acts as a superclass of another concept "turn on power at circuit breaker.'

- To represent the 355 hazard concepts, the aforementioned seven secondary classes were adapted from the OI\&ICM and, if applicable, their subclasses were used. Other intermediate classifications were also used in representing the hazard concepts. Some of the intermediate classifications were formed by some of the hazard concepts. For example, the concept "loss of load" is categorized as a subclass of the transportation accidents class and then also acts as a superclass of another concept "loss of load during transport." The other intermediate classifications were defined independent of these hazard concepts. For example, eight additional classes were defined, including action events/exposures and equipment events/exposures, under the secondary class other events or exposures to further categorize those hazard concepts belonging to that secondary class.

Another important task in validating the concept representation model was to represent relations between concepts through association and logical relationships. The following summarizes the relationship representation process in the test cases:

- Association relationships are established to connect activity to job step concepts and connect job step to hazard concepts.

- Disjoint relationships are only deployed to some activity and hazard concepts. There are 18 sets of multiple disjoint hazard concepts and one set of two disjoint activity concepts in the test cases. For example, the extracted hazard concepts "fall from rolling scaffold" and "fall from suspended scaffold" are declared disjoint.

- Equivalent relationships are deployed in two sets of two hazard concepts, which are "slip" and "trip" and "sprain" and "strain". All the required relations between the extracted concepts in the test cases were established through association and logical relationships.

\section{Case Studies for Validating the Document Representation Model}

The effectiveness of using the developed document representation model to represent the 78 acquired JHA documents was tested. The following examples are part of the implementation for the document representation with which the model's capability of representing documents in different representation scenarios was demonstrated.

- The representation of activity knowledge starts with representing activity title followed by the representation of applicability condition and job step knowledge, which is discussed in the next scenario. The applicability condition is described through a single activity concept.

- The representation of job step knowledge starts with representing job step title followed by the representation of applicability condition and hazard knowledge, which is discussed in the next scenario. The applicability condition is described through both activity and job step concepts. Two different subscenarios are found in the case studies. First, job step knowledge contains only a single job step and in these cases, a job step concept is sufficient, together with an activity concept, to describe the applicability condition. The other subscenario is that job step knowledge contains several job steps at the same time. In these cases, multiple job step concepts are represented together with an activity concept to describe the applicability condition of the job step knowledge.

- The representation of hazard knowledge starts with representing a hazard title followed by the representation of applicability condition and recommended safety procedure/safety rules. The applicability condition is described through activity, job step, and hazard concepts. The safety rules are represented through listing all safety rules corresponding to the activity, job step, and hazard concepts. Hazard knowledge contains either a single hazard or several hazards associated with a job step, both of which can be represented in a way similar to that used for job step knowledge.

\section{Summary of and Discussion on the Validation of the Representation Model}

The concept representation model enabled representation of all the extracted JHA concepts according to the proposed modeling strategies. It also enabled the establishment of necessary relations between the extracted concepts through the defined association relationships and logical relationships. On the other hand, the 
document representation model enabled representation of all the acquired JHA documents. The document representation model represented applicability conditions in multiple representation levels by interlacing uses of the concept conjunction tag $<$ Concept_AND $>$ and disjunction tag $<$ Concept_OR $>$, which enabled the document representation model to take into account complicated conditions engineers may encounter in their projects. Therefore, the capability of the concept and document representation models to respectively represent JHA concepts and JHA documents is successfully validated.

Both the concept and document representation models have representation limitations: when the number of concepts or documents becomes large, modeling these concepts in a concept ontology or documents in XML format will become tedious because the modeling processes must be performed manually. For concept representation, the limitation may be addressed through providing tools that allow a more straightforward process of modeling concepts and relationships between the concepts. For document representation, to address the limitation, using an editor that, for example, can automatically generate XML tags for users to fill in with required information is a better alternative than using a simple text editor. In addition, the use of an editing tool that provides user interfaces for directly entering information, such as those developed in the prototype system JHA Adviser, rather than using markup tags also is an option to address the limitation.

\section{Case Studies for Validating the Reasoning Mechanism}

This section presents the case studies performed to validate the effectiveness of using the reasoning mechanism to evaluate the applicability of JHA concepts and JHA knowledge. The discussions on these case studies are separated into two parts: case studies for the concept evaluation mechanism and for the document evaluation mechanism.

\section{Case Studies for Validating the Concept Evaluation Mechanism}

The concept evaluation mechanism to given conditions was validated by conducting several synthetic test cases. In these test cases, synthetic conditions were specified to test whether the developed concept evaluation principles could properly propagate applicability values between concepts and also identify concepts' applicability to the specified synthetic conditions. The concept evaluation mechanism was implemented in JHA Adviser, so the test cases conducted were also used to evaluate whether JHA Adviser can function properly to implement the evaluation process.

The following discusses three test cases, which are part of the tests for the validation and are representative of demonstrating the evaluation mechanism's capability.

Case Study 1. In this case, the following condition was specified: "A construction project is planned to use support systems during the upcoming excavation activity to prevent cave-ins." The activity concept "excavation using support systems" of the concept ontology can best describe the given condition. Therefore, this concept was assigned the applicability value of applicable. After that, the evaluation mechanism successfully evaluated the concept's primary and secondary classes, activity and excavation activity respectively, and its associated job step concept, "excavation using shoring manufactured trench boxes or other support systems," to be applicable. The evaluation mechanism also successfully evaluated the 11 hazard concepts associated with the abovementioned job step concept to be applicable. In addition, the mechanism evaluated this concept's disjoint concept, "excavation using sloping," to be not applicable.
The study result shows that the first, second, and fourth concept evaluation principles worked properly to propagate the applicability value from the concept "excavation using support systems" to its superconcepts and associated concepts.

Case Study 2. In this case, the following condition was specified: "There is no HVAC activity other than a plumbing activity scheduled for today." According to this description, the concepts best describing the situation were two activity concepts: "HVAC activity" and "plumbing activity." Therefore, the applicability value of not applicable was assigned to the former concept and the value of applicable to the latter concept. Then the evaluation mechanism successfully evaluated all the three subconcepts of "HVAC activity" to be not applicable. Also, the evaluation mechanism successfully evaluated the primary class activity of the concept "plumbing activity" to be applicable.

Because both concepts "HVAC activity" and "plumbing activity" were secondary classes and only used for classification, no association relationships exist between them and other job step concepts. The result shows that the first concept evaluation principle worked properly to propagate both the applicability values applicable and not applicable in class hierarchies.

Case Study 3. In this case, the following condition was specified: "Some accidents, including workers' sprain, and slip and fall due to poly moving, and being struck by a truck, happened yesterday on the project's construction site." According to this description, the concepts best describing the condition were four hazard concepts: "sprain," "slip due to poly moving," "fall due to poly moving," and "struck by." Hence, the applicability value of applicable was assigned to all of these concepts. After that, the evaluation mechanism successfully evaluated these concepts' primary and secondary classes and classes of other intermediate classifications to be applicable. For example, the superclasses of the concept "slip due to poly moving," potential hazard, hazard from bodily reaction and exertion, bodily reaction, and slip, were found applicable. Moreover, the equivalent concept of the concept "slip," "trip," was then evaluated to be applicable. Similarly, the equivalent concept of the concept "sprain," "strain," was also successfully evaluated to be applicable.

The test case result shows that the first and third concept evaluation principles worked properly to propagate the applicability value applicable in class hierarchies and to equivalent concepts.

\section{Summary of and Discussion on the Validation of Concept Evaluation Mechanism}

The test cases discussed in this section demonstrate that the concept evaluation mechanism and prototype system JHA Adviser are capable of correctly evaluating concepts' applicability in the given testing scenarios. In addition to those concepts describing the synthetic conditions, other concepts represented in the concept ontology were also tested. Expected evaluation results were also obtained once the necessary association, disjoint, and equivalent relationships were properly defined between the concepts. Therefore, the concept evaluation mechanism can be considered validated in its ability to correctly evaluate JHA concepts that are modeled in an ontology.

To make evaluating concepts manageable, only singleinheritance was allowed in the concept ontology. Removing this limitation and allowing multiple-inheritance in the concept ontology reasoning mechanism is worth further study in future research.

\section{Case Studies for Validating the Document Evaluation Mechanism}

The document evaluation mechanism was validated through several test cases. In these test cases, conditions were specified to test 
whether the document evaluation rules could properly propagate applicability values from the concepts to the represented JHA documents and to test whether all JHA safety rules related to the specified conditions/concepts could be correctly evaluated to be applicable and/or not applicable. The document evaluation mechanism was implemented in JHA Adviser, so the test cases conducted were also used to evaluate whether JHA Adviser implemented the evaluation processes properly.

The validation of the document evaluation mechanism started by assigning applicability values to single concepts. Then multiple concept tests were conducted in which applicability values were assigned to multiple concepts.

Single concept tests. This scenario tested how well the reasoning mechanism functioned when assigning applicable or not applicable value to a single concept.

Assign applicable value to single concept. The value of applicable was assigned to some of the represented concepts, which were selected from the three primary classes of the concept ontology to observe whether all the relevant applicable or not applicable JHA safety rules were correctly evaluated and identified. The test results are shown in Table 3.

The cases in Table 3 demonstrate the tests on assigning applicable value to activity, job step, and hazard concepts. For the test case Numbers 5 to 12, because the assignment of applicability value was not on activity concepts, the concept neglect mechanism was involved in these test cases to ensure the reasoning mechanism's proper functioning. Activity concepts were all neglected in test case Numbers 5 to 8 , and both activity and job step concepts were neglected in test case Numbers 9 to 12 .

The results of these tests show that the document evaluation mechanism, except for test case Number 8 , successfully identified applicable JHA safety rules and not applicable JHA safety rules, which were only available in the test cases when there were disjoint relationships in the evaluation processes. The reason why the applicable JHA safety rules were not identified in the test case Number 8 is that multiple job step concepts were used to describe the applicability condition. Therefore, only assigning the applicable value to one of the job step concepts was not sufficient to evaluate the corresponding JHA safety rules' applicability value.

Assign not applicable value to a single concept. The evaluation of assigning the not applicable value to a single concept can be illustrated using those test cases in Table 3 in which disjoint relationships were involved because disjoint relationships help determining not applicable concepts based on applicable ones. For example, specifying the test concept applicable in test case Number 2 in Table 3 makes another activity concept "excavation using slopes" not applicable due to the disjoint relationship. The document evaluation mechanism then successfully identified the not applicable safety rules for this not applicable activity concept. As shown in Table 3 (case Numbers 2, 6, 8, and 10), when a single concept is automatically given not the applicable value through reasoning over disjoint relationships, not applicable safety rules can be properly evaluated and identified.

Multiple concept tests. This scenario tested the document evaluation mechanism by assigning applicable and/or not applicable value to multiple concepts. For the convenience of discussion, two activity concepts in each test case are used in the following scenarios.

Assign applicable value to multiple concepts. Two representative test cases were conducted to demonstrate this subscenario. In the first case, two activity concepts "frame columns" and "excavation using support systems" were assigned the applicable value. After the evaluation processes, the evaluation results show that the applicable and not applicable safety rules were correctly identified when these two concepts' applicabilities were taken into account together, as shown in the test cast Number 1 in Table 2. In addition, the numbers of applicable and not applicable safety rules in the combined evaluation process (i.e., 49 and 23) are equal to the sum of the numbers of applicable and not applicable safety rules in the separate evaluation process, respectively. This means evaluating the two concepts are independent processes, i.e., one process does not affect the other.

In the second case, another two activity concepts "forklift use" and "decontamination of windows" were assigned an applicable value. It was also observed that the applicable and not applicable safety rules were correctly identified in this case after the evaluation processes, as shown in the test case Number 2 in Table 2. However, the number of not applicable safety rules in the combined evaluation is different from the sum of the numbers of those in the separate evaluation. This indicates that there were concepts whose applicability values were updated due to applicability contradictions during the process of evaluating the second concept "decontamination of windows."

Table 3. Test Case Results of Assigning Applicable Value to a Single Concept

\begin{tabular}{|c|c|c|c|c|c|}
\hline Test case & Test concept & Primary class & Secondary class & $\begin{array}{l}\text { Applicable safety } \\
\text { rules identified }\end{array}$ & $\begin{array}{l}\text { Not-applicable safety } \\
\text { rules identified }^{\mathrm{a}}\end{array}$ \\
\hline 1 & Frame columns & \multirow{4}{*}{ Activity } & Concrete activity & True & N/A \\
\hline 2 & Excavation using support systems & & Excavation activity & True & True \\
\hline 3 & Working on or near energized circuit & & Electrical activity & True & N/A \\
\hline 4 & Install process piping & & Plumbing activity & True & N/A \\
\hline 5 & Access mobile scaffold & \multirow{4}{*}{ Job step } & Access step & True & N/A \\
\hline 6 & \multirow{3}{*}{$\begin{array}{c}\text { Check dimension with proper machining tools } \\
\text { Cutting off tube } \\
\text { Raise chuck guard }\end{array}$} & & Check step & True & True \\
\hline 7 & & & Cut step & True & N/A \\
\hline 8 & & & Raise step & False & True \\
\hline 9 & Strain & \multirow[b]{3}{*}{ Hazard } & Bodily reaction and exertion & True & N/A \\
\hline 10 & Injury to personnel due to hydraulic jack crushing & & Contact with objects and equipment & True & True \\
\hline 11 & Exposure to radiological contaminants & & $\begin{array}{c}\text { Exposure to harmful } \\
\text { substances or environments }\end{array}$ & True & N/A \\
\hline 12 & Fall into excavations & & Falls & True & N/A \\
\hline
\end{tabular}

${ }^{\mathrm{a}} \mathrm{N} / \mathrm{A}$ indicates that there are no not-applicable safety rules identified for the test concept. 
Assign not applicable value to multiple concepts. When JHA concepts are assigned a not applicable value, no concepts will be deduced applicable according to the concept evaluation principles. Therefore, if the concepts that were assigned a not applicable value were used to describe the applicability conditions and were incorporated in logical AND, the JHA safety rules with the applicability conditions will be evaluated not applicable according to the document evaluation rules.

The test case Number 3 in Table 2 proves this evaluation argument. Two activity concepts "carcinogen control" and "spray painting" were assigned a not applicable value. 27 not applicable safety rules were successfully identified. No safety rules were evaluated applicable, as expected.

Assign both applicable and not applicable values to multiple concepts. This subscenario is a combination of the previous two. The result of a test case conducted for this subscenario is listed as test case Number 4 in Table 2, in which an activity concept "frame columns" and one of its job step concepts "set pins" were respectively assigned applicable and not applicable values. The result illustrated that both applicable and not applicable safety rules were identified by the document evaluation mechanism. That is, the document evaluation mechanism is also proved to function properly in evaluation concepts belonging to this subscenario.

\section{Summary of and Discussion on the Validation of Document Evaluation Mechanism}

Several test cases were discussed to prove that the document evaluation mechanism is capable of evaluating JHA safety rules to be applicable or not applicable. The evaluated safety rules can be successfully classified according to their applicability, and the classified rules are output into a Microsoft Excel spreadsheet file using JHA Adviser.

In the scenario of assigning an applicability value to a single concept, the document evaluation mechanism is only not successful in identifying an applicable safety rule when the safety rule's applicability condition is described through several job step or hazard concepts and the concept being assigned a value is just one of them-therefore sufficient information is lacking to determine the applicability of the safety rule.

In the scenario of assigning applicability values to multiple concepts, evaluation processes are performed in turns (one process will not start until the previous one is finished), and the evaluation results are cumulative. Cumulative evaluation means the safety rules, applicable or not applicable, identified in a later evaluation process can join those in earlier evaluation to form the final set of safety rules. Examples of cumulative applicable safety rules are shown in test case Numbers 1 and 2 in Table 2, and examples of cumulative not applicable rules are shown in test case Numbers 1, 3, and 4 in Table 2. However, there is an exception to this cumulative feature: when different evaluation processes have common concepts whose applicability values change (from applicable to not applicable or vice versa) during evaluation, the results of evaluating safety rules will not be cumulative. An example of noncumulative applicable safety rules is shown in test case Number 3 in Table 2, and an example of noncumulative not applicable ones is shown in test case Number 2 in Table 2. Situations of noncumulative reasoning usually involve concept applicability contradictions, and users must resolve the contradiction before continuing the evaluation process.

One limitation of the document evaluation mechanism is observed during the validation: if there are redundant safety rules identified, i.e., safety rules with exactly the same content, the evaluation mechanism currently does not remove redundancies. Although this limitation does not affect the correct operation of the reasoning mechanism, it may lessen the readability of identified safety rules when the number of the safety rules becomes larger This issue is expected to be addressed in future research.

\section{Conclusions}

This research proposed a framework to help improve access to a company's JHA knowledge. The framework adopts ontological modeling to organize JHA concepts of JHA knowledge and document modeling to organize JHA documents in XML format. XML provides a human-readable and well-structured format; therefore, it allows safety rules to be managed easily and enables automated reasoning about the applicability of safety rules. The framework also provides systematic reasoning mechanisms to help evaluate the applicability of JHA concepts and identify applicable JHA safety rules.

JHA safety rules can be tied to concepts of activities, job steps, and/or hazards in the framework, and this ensures the applicable safety rules can be better identified. For example, to reason about safety rules and identify the rules associated with the input concepts, engineers can use only one type of the concepts, e.g., hazards, to describe a construction applicability condition when other concepts, e.g., activities or job steps, are unknown or cannot be properly identified. From the case studies, it was found that the proposed framework and the developed prototype system JHA Adviser were able to identify applicable JHA safety rules from the JHA knowledge (documents). Expected results are successfully attained in the various test cases conducted in this research. The proposed framework is expected to be useful because engineers can use it to more easily conduct new JHAs, prepare new JHA documents, or retrieve previous JHA knowledge.

According to experienced safety engineers' feedback, the advantage of the proposed framework over the traditional JHA approach is twofold. First, they can more easily use and retrieve JHA knowledge and spend less time on determining what concepts should be considered in new JHAs and preparing new JHA documents. Second, they can more quickly react to context changes on site and generate new JHA documents for the new contexts by selecting different concepts that best describe the contexts. The safety engineers also suggest that the proposed framework can be further improved by integrating construction safety specifications, such as the OSHA safety regulation for construction safety (U.S. Department of Labor 2003), with JHA knowledge to give engineers more information about safety requirements. We aim to address these issues in the proposed framework in future research. In addition, the proposed framework needs further studies to remove the two limitations observed and discussed in the case studies: singleinheritance in the concept evaluation mechanism and possible redundancy of safety rules in the document evaluation mechanism. This upgrade can provide engineers more supportive safety information and benefit their safety planning and decision making. Finally, additional research will be required to integrate the proposed framework with building information modeling tools to enable a more automated construction context identification, as proposed in an earlier publication (Wang and Boukamp 2007)

\section{Acknowledgments}

The authors would like to thank Patrick McGowan, Daniel Ruane, and James Smith from W. E. O'Neil Construction of Chicago for sharing their valuable knowledge and supporting this research. 


\section{References}

Abdelhamid, T. S., and Everett, J. G. (2000). "Identifying root causes of construction accidents." J. Constr. Eng. Manage., 126(1), $52-60$.

Akinci, B. (2000). "Automatic generation of work spaces and analysis of time-space conflicts at construction sites." Ph.D. tesis, Stanford Univ., Palo Alto, CA.

Antoniou, G., and van Harmelen, F. (2004). A semantic web primer, MIT Press, Cambridge, MA.

Arboleda, C. A., and Abraham, D. M. (2004). "Fatalities in trenching operation-Aalysis using models of accident causation." J. Constr. Eng. Manage., 130(2), 273-280.

Aziz, Z., Anumba, C. J., Ruikar, D., Carrillo, P. M., and Bouchlaghem, N. M. (2005). "Context aware information delivery for on-site construction operations." 22nd CIB W78 Conf. on Information Technology in Construction, International Council for Research and Innovation in Building and Construction, Rotterdam, Netherlands, 321-332.

Bechhofer, S., et al. (2004). "OWL web ontology language reference." 〈http://www.w3.org/TR/owl-ref/〉 (Sep. 1, 2009).

Breitman, K. K., Casanova, M. A., and Truszkowski, W. (2006). Semantic web: Concepts, technologies and applications, 1st Ed., Springer, London.

Chen, H., Finin, T., and Joshi, A. (2003). "Using OWL in a pervasive computing broker." 2nd Int. Conf. on Autonomous Agents and Multiagent Systems, International Foundation for Autonomous Agents and Multiagent Systems, Richland, SC, 9-16.

Construction Specifications Institute. (2005). "MasterFormat 2004 Edition." Alexandria, VA.

Darlington, M. J., and Culley, S. J. (2008). "Investigating ontology development for engineering design support." Adv. Eng. Inform., 22, $112-134$.

DeRose, S. J., Durand, D. G., Mylonas, E., and Renear, A. H. (1990). "What is text, really?" J. Comput. Higher Educ., 1(2), 3-26.

Dolenc, M., Katranuschkov, P., Gehre, A., Kurowski, K., and Turk, Ž. (2007). "The InteliGrid platform for virtual organisations interoperability." J. Inf. Technol. Constr., 12, 459-477.

El-Diraby, T. E., Lima, C., and Feis, B. (2005). "Domain taxonomy for construction concepts: Toward a formal ontology for construction knowledge." J. Comput. Civ. Eng., 19(4), 394-406.

Gruber, T. R. (1993). "A translation approach to portable ontology specifications." Knowl. Acquis., 5(2), 199-220.

Harold, E. R., and Means, W. S. (2004). XML in a nutshell, O'Reilly, Sebastopol, CA.

Hinze, J., Pederson, C., and Fredley, J. (1998). "Identifying root causes of construction injuries." J. Constr. Eng. Manage., 124(1), 67-71.

Horridge, M., Knublauch, H., Rector, A., Stevens, R., and Wroe, C. (2009). "A practical guide to building OWL ontologies using the protégé-OWL plugin and CO-ODE tools."〈http://owl.cs.manchester.ac.uk/tutorials/
protegeowltutorial/resources/ProtegeOWLTutorialP4_v1_2.pdf $\rangle$ (May 30, 2010).

Kim, C., Haas, C. T., Liapi, K. A., and Caldas, C. H. (2006). "Humanassisted obstacle avoidance system using 3D workplace modeling for construction equipment operation.” J. Comput. Civ. Eng., 20(3), 177-186.

Kim, E., and Choi, J. (2006). "An ontology-based context model in a smart home." Int. Conf. on Computational Science and Its Applications, Springer, Berlin, 11-20.

Lima, C., El-Diraby, T. E., and Stephens, J. (2005). "Ontology-based optimization of knowledge management in e-construction." J. Inf. Technol. Constr., 10, 305-327.

Navon, R., and Kolton, O. (2007). "Algorithms for automated monitoring and control of fall hazards." J. Comput. Civ. Eng., 21(1), 21-28.

Protégé. (2008). "Protégé."〈http://protege.stanford.edu〉 (Aug. 27, 2008).

Renear, A. H. (2008). "Descriptive markup and the OHCO model of text." Document modeling, Graduate School of Library and Information Science, Univ. of Illinois, Champaign, IL.

Rezgui, Y. (2006). "Ontology-centered knowledge management using information retrieval techniques." J. Comput. Civ. Eng., 20(4), 261-270.

Roughton, J. E., and Crutchfield, N. (2007). Job hazard analysis: A guide for voluntary compliance and beyond, Butterworth-Heinemann, Oxford, UK.

U.S. Bureau of Labor Statistics. (2007). "2006 census of fatal occupational injuries." Washington, DC.

U.S. Dept. of Labor. (1992). "Occupational injury and illness classification manual." Bureau of Labor Statistics, Washington, DC.

U.S. Dept. of Labor. (2002). "Job hazard analysis." Publication 3071, Occupational Safety and Health Administration, Washington, DC.

U.S. Dept. of Labor. (2003). "Occupational Safety and Health Standards for the Construction Industry." 29 CFR 1926, Occupational Safety and Health Administration, Washington, DC.

W3C. (2004a). "OWL: Web ontology language."〈http://www.w3.org/ 2004/OWL/> (May 1, 2010).

W3C. (2004b). "XML schema." 〈http://www.w3.org/XML/Schema〉 (May $1,2010)$

Wang, H.-H. (2010). "A context-based representation and reasoning formalism to support construction safety planning." Ph.D. dissertation, Univ. of Illinois, Urbana, IL.

Wang, H.-H., and Boukamp, F. (2007). "Leveraging project models for automated identification of construction safety requirements." Int. Workshop on Computing in Civil Engineering, ASCE, Reston, VA.

Wang, H.-H., and Boukamp, F. (2008). "A context ontology development process for construction safety." Joint CIB Conf.: W102 Information and Knowledge Management in Building and W096 Architectural Management, International Council for Research and Innovation in Building and Construction, Rotterdam, Netherlands, 297-308.

Wang, X. H., Zhang, D. Q., and Gu, T. (2004). "Ontology based context modeling and reasoning using OWL." 2nd IEEE Annual Conf. on Pervasive Computing and Communications Workshops, IEEE, New York. 